\title{
Wireless Sensor Networks for Road Traffic Monitoring
}

\author{
Kahtan Aziz \\ College of Engineering Computing. \\ Al Ghurair University \\ Dubai, United Arab Emirates
}

\author{
Salah Haj Ismail \\ Aleppo University \\ Aleppo, Syria
}

\author{
Saed Tarapiah \\ Telecommunication Engineering Dept. \\ An-Najah National University Nablus, \\ Palestine
}

\author{
Mohanad Alsaedi \\ Amarah Gas Power Plant Project \\ Amarah, Iraq
}

\begin{abstract}
Wireless Sensor Networks (WSNs) consist of large number of sensor nodes. Each node is empowered by a communication interface that is mainly characterized by low power, short transmission distance and minimal data rate such as the maximum data rate in ZigBee technology is $256 \mathrm{kbps}$, while approximately the physical transmission range between 10 to 20 meters. Currently, WSN Technology is being distributed over a large roadway of areas, in order to monitor traffic and environmental data. This approach allows several Intelligent Transport Systems (ITSs) applications to exploit the primary collected data in order to generate intelligent decisions based on earlier valuable selected information. Therefore, in this work we present a MAC protocol that is suitable for WSN where its nodes are assigned to a linear topology. The investigated protocol is realized by adapting an already existing Jennic MAC protocol. We demonstrate the validity of the MAC by building a complete end-to-end road traffic monitoring system using 4 Jennic nodes deployed in an indoor environment with the aim to prove the MAC potential in meeting the expectations of ITS applications. It is appropriate to mention that the proposed implementation just considers stationary WSN nodes.
\end{abstract}

Keywords-Wireless Sensor Networks(WSN); Linear Topology; Road Monitoring; Jennic MAC

\section{INTRODUCTION}

Intelligent Transportation Systems (ITSs) starts to receive much attention recently by research institutes, industrial factories and standardization entities as they affect widely the life of people. The scope of ITSs is to provide fundamental services and applications that will improve transportation and mobility safety and enhance the available resources and time which influences on driving speed and affects crash rates. ITS services and applications depend on deploying advanced technologies and distributing them on the intelligent infrastructure systems and vehicles systems. These advanced technologies consists of physical world perceive technologies that are able to provide real world measurements and convert them into the digital world, storage capabilities to work on the digital measurements which will save and analyse these data and the communication technologies of both wired and wireless technologies to exchange the collected data with the vehicles and their infrastructure and vice versa [1] [2].

A system that monitors and reports the physical condition of roads such as slipperiness factor, humidity and road works ... etc; and estimates traffic on different road segments would provide very useful information for further analysis and decision that would be taken or generated by the system. Information generated from this system could be integrated with SMS based services that alert users about congestion, automatic traffic light timers, geographic information systems that suggest less congested paths or roads which are less damaged, systems that trigger road maintenance work and analysis tools that help to manage traffic and plan extensions to the road network, which will enhance the efficiency of traffic circulation, minimize risks and time to take corrective actions both on design and management levels. There are several challenges in building such a system; Such challenges fall in the areas of sensing, signal processing, communication links, protocol design, information storage and retrieval. Traffic on the road or condition of the road can only be determined through some dedicated and in purpose designed sensors.

Road traffic scenarios change dynamically, where many previous studies have been conducted in order to characterize the dynamic road traffic scenario [3] and the response to congestion must be swift. Therefore, communication protocol for such a system must be near real time to provide meaningful and useful data.

Traffic monitoring systems generate huge amount of data where the systems by its turn must process these useful information, especially those systems that need historical information to correctly estimate current state of traffic.

Traffic monitoring systems generally try to count, classify or estimate speed of vehicles moving on the road.

It is clear that a road condition and traffic monitoring system must be economically feasible so some tunning to the system can be applied such as reducing the number of system components and reusing and utilizing existing infrastructure.

The system should be feasible and able to avoid the need of digging up roads or creating additional infrastructure in form of 
laying wires or making overhead structures. It should be built using cheap and easily available commercial. The aim of this work is to build a road monitoring system that is able to better quantify a road anomaly. To achieve such goal we propose an approach to improve road monitoring through wireless sensor networks (using Jennic jn-5148 wireless sensors by NXP) to build a wireless sensor network where several sensors distributed along a road in forced linear topology.

\section{RELATED WORK}

The early works, [4] and [5], can be considered of earliest works that sought to provide a traffic monitoring application based WSN concepts and technology rather than relying on traditional methods. However, the authors of these two works showed a sophisticated MAC protocol which is divided into two parts. The first part is suited for sensor nodes deployed under surface of pavements along sides of the roads. The second part is designed for access points. Furthermore, the overall resulted network protocol supports bidirectional communication links. The first link is to transfer collected and sensory readings from the sensor nodes to the access point via multi-hop fashion. While the later link is used to carry data packets and synchronization commands from the reverse direction (from the access point to sensor nodes) in single hop manor. Such approaches leads to asymmetric communication between sensor nodes and access points in order to cope with the difference in their communication range such property inconsistent with our assumption that all the nodes have similar communication capabilities and these two works are suitable for ITS applications that is using WSN deployed in star topology not for Linear topology.

The authors of the earlier paper [6] presents a brief survey of the important aspects for LWSN namely its applications as well as issues. Mainly, the paper classifies LWSN topologies into three main categories thin, thick and very thick. The thin structure applies where all sensor nodes are physically deployed in a line.

This section discusses the primary MAC implementations that are supported by the Jennic sensor nodes. Which is the most suitable to be considered and integrated in the proposed approach (Namely IEEE 802.15.4, ZigBee and Jennic proprietary MAC). At the end of this section, a general discussion of related research work are provided.

Supported MAC Implementations are : I) IEEE 802.15.4 is a standard protocol that builds the physical layer (PHY) and Media Access Control layer (MAC). This standard is suitable for low power, short range and low bit rate in particular the Wireless Personal Area Networks (WPAN). The JenNet and ZigBee Pro (will be explained briefly bellow) are built on top of IEEE 802.15.4 implementation while keeping the ability to develop a novel application through interfacing it directly with the IEEE 802.15.4 API (discarding the JenNet and ZigBee Pro implementations). II) JenNet is a proprietary network protocol implemented by Jennic Network [7]. JenNet aims to simplify the development of new application on top of Jennic wireless devices through providing the implementation of full network layer - the third layer on the OSI model-. Actually, JenNet runs on top of IEEE 802.15.4 standard implementation. JenNet is supplied with an API, known as the Jenie API, to facilitate the

\begin{tabular}{|l|l|l|l|}
\hline Feature & IEEE 80.2.15.4 & JenNet & ZigBee Pro \\
\hline $\begin{array}{l}\text { Supported } \\
\text { Topologies }\end{array}$ & Point-to-Point & $\begin{array}{l}\text { Tree } \\
\text { Star } \\
\text { Line }\end{array}$ & Mesh \\
\hline $\begin{array}{l}\text { Maximum } \\
\text { Number of } \\
\text { nodes }\end{array}$ & 50 nodes & 500 nodes & 50 nodes \\
\hline $\begin{array}{l}\text { Third Party In- } \\
\text { teroperability }\end{array}$ & no provisioning & $\begin{array}{l}\text { no provision- } \\
\text { ing }\end{array}$ & $\begin{array}{l}\text { Interoperability ZigBee } \\
\text { through and } \\
\text { public profiles and } \\
\text { compliance/certification }\end{array}$ \\
\hline Licensing Cost & Free & Free & $\begin{array}{l}\text { ZigBee Alliance mem- } \\
\text { bership and product cer- } \\
\text { tification }\end{array}$ \\
\hline
\end{tabular}

TABLE I: supported MAC implementations feature Comparison

interaction between the application and the JenNet stack. III) ZigBee Pro is an industry-standard protocol where the two protocols are implemented on top of the IEEE 802.15.4 protocol. Zigbee Pro provides the network stack implementation of the OSI reference model in order to facilitate the implementation of new wireless network application developments. Zigbee Pro and JenNet Operating System (OS) API are used to realize new application for Jennic devices.

We concisely present the supported MAC implementations in the properties, which are most relevant to this work comparison in terms of, Application field, Technology, Topology, MAC, Sensor, and Energy. We emphasize that JenNet and ZigBee Pro are using IEEE 802.15.4 as the foundation to build end-to-end WSN applications. We include IEEE 802.15.4 here to form a baseline.

A survey on the different mac layer protocols for linear wireless sensor networks is further discussed in [8]

\section{LineAR TOPOLOGY MAC PROTOCOL DESIGN}

The implementation of the proposed protocol just considers a network which is a linear topology for wireless sensor network (LWSN). Such network consists of low power nodes connected via wireless links arranged on a single line. These nodes are located along the side of the road segments, as depicted in Figure 1. Usually, linear topologies exist on pipelines, rivers and railways. Many other possible candidate applications could be also considered as in [9], [10] and [11] not only for speed monitoring or temperature measurement, but also for GIS data collection to enhance the health of important structures as tunnels, bridges, and kinetic traffic structures to avoid disasters and improve environmental quality by measuring co2 emissions inside tunnels and ventilate it properly in times of congestion, vibration of bridges and avoid collapse caused by resonance,surface humidity and protect vehicles from slipping away by forcing proper speed ... etc. For such topology, ZigBee cluster-tree can be implemented as well to exchange data between nodes, where this method suffers from limitations in LWSN: the limited number of children, and as well as the maximum number of children routers, and the maximum tree depth as stated and discussed by [12]. Therefore, the goal of this work is to design a new MAC protocol that would take advantage of the linear topology nature and characteristics in order to minimize the energy consumption needed to exchange the collected date among the sensor nodes. 


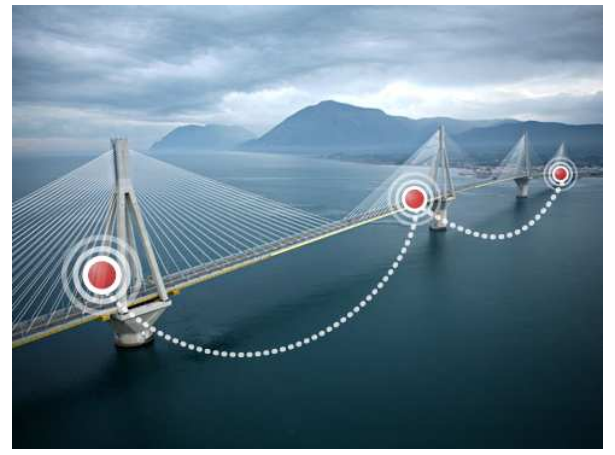

Fig. 1: wireless sensors placed on bridge [13]

Thus, several requirements have been taken into consideration.

\section{A. LWSN Node Types}

The nodes of LWSN based network are of the following general types, which classified based on their roles in the network:

I Personal Area Network (PAN) Coordinator: There must be one and only one PAN Coordinator. Where it performs the following network functions: a) Assigning a PAN ID (a unique 16-bit value) to the network. b) Selecting radio frequency to be assigned for network operation. c) Identify and assign a short address (a unique 16 bit value) to itself. d) Handling requests from other devices to join the network e) Relaying messages from one node to another.

II Router Node: A LWSN network can have one or more routers. Each of these routers serves its own children and its roles include:

a Handling requests from other devices to join the network.

b Relaying messages from one node to another.

III End Device: This is a node which has a sensor and/or actuator functions but no coordinating functionality. The term "End Device" is not used in the IEEE 802.15.4 standard, but is commonly used in the field (wireless sensor network).

For better understanding, we consider LWSN as depicted in Figure 2. Which consists of one node acts as the network PAN coordinator, one End Device while other nodes act as router nodes. the nodes of LWSN are arranged in a logical line.The start of the line is the PAN Coordinator and the end of the line is the End Device. The line sequence passes by order starting from one router node to the next router nodes until it reaches the End Device.

Therefore, to send a data packet from the Edge device to the network Coordinator, the message will be sent via router nodes, which relay the message to the next router node until it arrives the Coordinator node. This linear topology uses two type of packets (Downlink and Uplink), the Downlink packet is a request from gateway node (coordinator) to all nodes asking them to answer with Uplink packet with their current reading as shown in Figure 2.

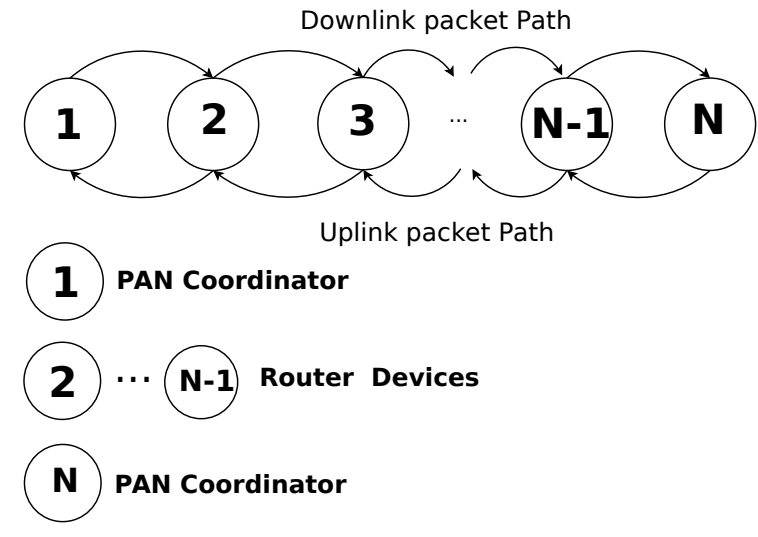

Fig. 2: LWSN Topology and type of network devices

\section{B. Nodes Addressing}

The Simple way to refer to a node in a network in the LWSN under study is by a numeric address. In JenNet, the MAC address which is a unique and permanent 64-bit value assigned by the manufacturer to a device is used which is also called an extended address. JetNet uses 64 bits address to identify a node in the network address.

\section{Network Protocol Stack}

The LWSN node software stack is illustrated in Figure 3. At the application level, the user applications that make use of the node provided services. The application that is defined by user works together with the network mainly via the Jenie API. Network Protocol Level is the JenNet network layer that controls network routing and addressing by calling functions in the IEEE 802.15.4 MAC layer.Network Protocol Level responsibilities are:

1) Initialize and setup the communication in the network.

2) controls joing and leaving the nodes of the network by allowing them to join or removing it.

3) Route messages to their planned routes.

4) grant secure communication link for the routed messages between the nodes of the network.

The Physical/Data Link Level. This Link Level is offered by the IEEE 802.15.4 standard and defined by two divided layers:

1) Data Link layer: This Link layer is offered by the IEEE 802.15.4 MAC layer. Where, the main MAC layer services are to provide reliable communication link at the MAC layer as well to provide fragmentation and reassemble of data. Moreover, MAC layer provides addressing by assigning Physical MAC Address to the data frames.

2) Physical layer: This layer is offered by the IEEE 802.15.4 PHY layer. It is working with the physical transmission medium interface, exchange data bits with this medium, and exchange data bits with the above layer (the Data Link layer).

The Jenie API offers the main strategy for the user application to work with the JenNet software stack. The API includes 


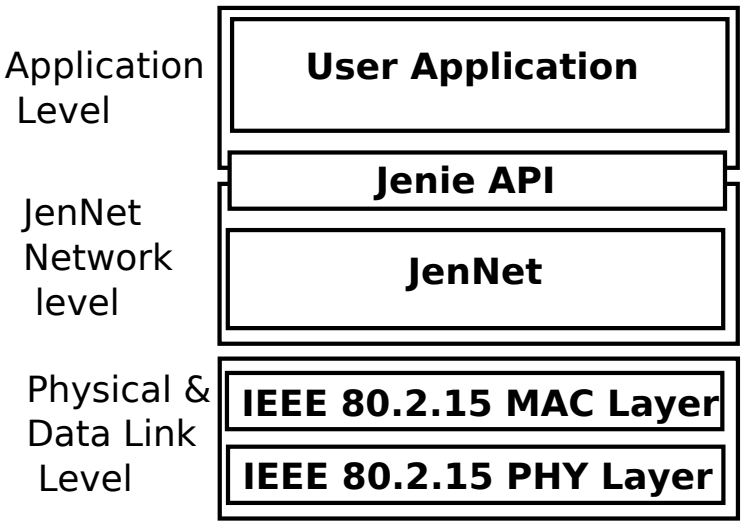

Fig. 3: Detailed Software and Network Stack for LWSN node

C functions and related resources (data types, enumerations, etc.), and provide a an easy-to-use and simple interface that is built to streamline the development of wireless networks applications. Finally, the JenNet API may be used in combination with the Jenie API to access the underlying JenNet stack layer features. The JenNet API includes $\mathrm{C}$ functions which provide extra rules where nodes can join a network, inter-network communication and the JenNet stack operation.

\section{END-TO-END APPLICATION IMPLEMENTATION}

To boost the implementation time and cost, the work uses JenNet application template as the base foundation for the Linear Topology MAC protocol. This template is provided by Jennic [7] and the details is in the Application Note JenNet Application Template (JN-AN-1061) [14].

Actually, the JenNet Template presents a good foundation for building a new JenNet applications. As an individual basic code is specified for each node type: Router, Coordinator and End Device. The developers of the embedded System can change the provided code to adapt it to their own application needs. The provided application templates consider the following:

1) the considered network topology is A Tree only.

2) Just one device is selected to be the PAN Coordinator.

3) one other device At least (to act as an End Device Or Router).

4) The Network Application ID and the PAN ID have a pre-identified values.

In fact the last 3 assumed points by the Jennic Template fits perfectly with the works engineering requirements. While, the first point (a Tree topology) needs to be redesigned and to be implemented in order to fit with the desired application and linear topology. Thus, paper main contribution is to provide the design guideline and implementation methods to generate a valid linear topology.

The main methods can be applied to generate a linear topology lay on the following ways:

1) Physical Distance to force a Linear topology in LWSN: In the Jennic template the linear topology is part of tree topology but the constrain is the

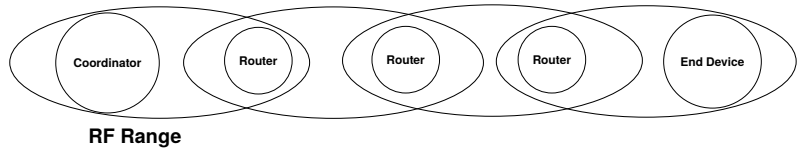

Fig. 4: Detailed Software and Network Stack for LWSN node

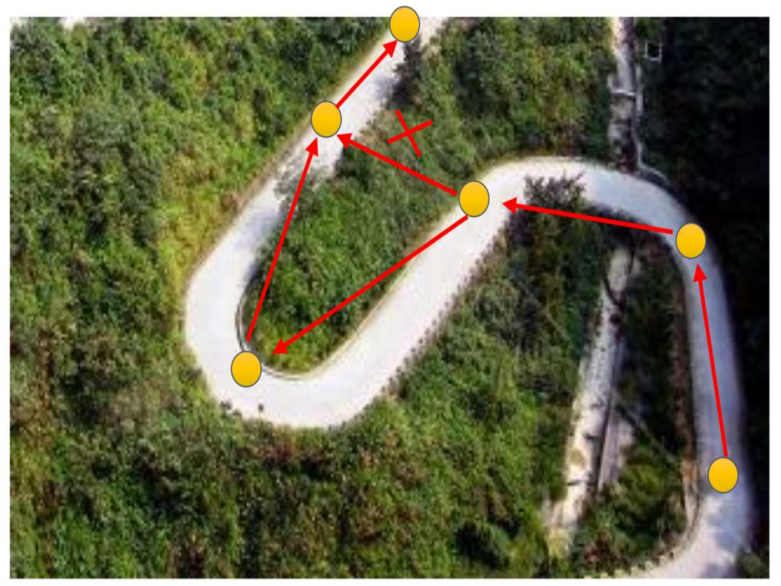

Fig. 5: WSN in Practical situation where RF range of nodes overlap randomly

distance i.e. if an engineer wants to have a linear topology he/she must arrange the network nodes in linear way and assures that just one node resides in the Radio Frequency (RF) range of another node figure 4. This method suffers from major problem when the nodes are near each other (this is common practical situation). In this case the resulted topology will change to another topology may be tree topology or any other topology. For further details, Figure 5 illustrates practical case of the problem.

2) Higher Network Layer to force a Linear topology in LWSN: This method resolves the later described using distance based method problem. The proposed method is to modify the network stack code to make sure that the resulted network topology is a linear one, this method works always even if nodes are in same RF range. The following steps are used to force Linear Topology: re-implementing the Authority function [14]: to accept or reject any node based on configuration file deployed in each node. The configuration file in a node includes list of the nodes' addresses to be connected to the node hosting the file, any unknown node (not in the addresses list) will be rejected. This method ensures always that the resulted topology is linear one as requested by the work engineering requirements.

\section{EXPEREMENTS AND MEASUREMENTS}

This section details preliminary experiments performed to validate the proposed methods and it includes the collected results to show the network performance. In these experiments all the 3 types of network nodes are included, PAN coordinator node, router nodes and End Device. All the experiments are 


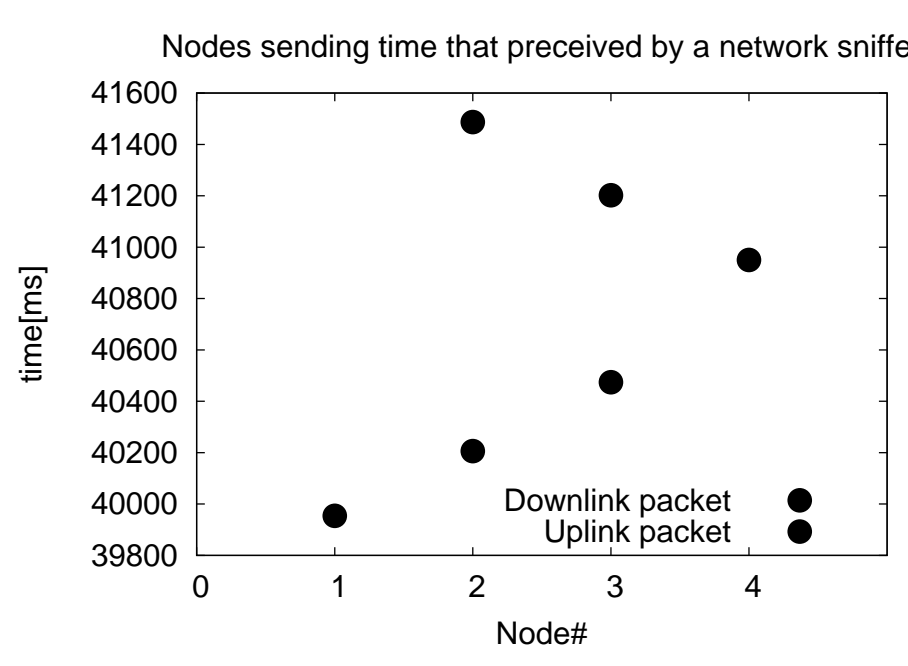

Fig. 6: Performance Evaluation

carried out with Jennic JN5148-EK010 Evaluation KIT [15]. The End-to- End application scenario represents a road monitoring system, where the road administrative authorities monitor the road environment temperature and the network nodes battery levels in real time manor. Such information could be visualized and controlled using a web application remotely, and furthermore, have the ability to control the lights appliances that are installed in both sides of the road.

\section{A. Experiments with LWSN of 4 Nodes}

The LWSN built with 4-nodes, after the network starts. The coordinator node sends Downlink request with address $0 x F F F F F F F F$ and specify the required sensor value,in this case the temperature readings data as well as the battery voltage data sensors. Measuring the LWSN Round Trip Time (RTT): $R T T=$ The time of Coordinator start sending Downlink packet until receiving the corresponding Uplink packet from the End Device. For this the 4 nodes setup where distance spacing between the nodes are identical and is equal 1 meter, the average RTT values is 1.533 seconds.

Figure 6 shows, the measured time in millisecond, observed by a network sniffer deployed in the radio frequency range of all the running network nodes, the measured time increases linearly with distance from node to coordinator node. The packet flow starts by initiating a packet request, DownLink packet, from the coordinator, where each packet received and processed by all the router nodes until it arrives to the End device. Which in turn, start a new uplink packet in response. The response packet will include the End Device temperature and voltage readings and forwarded to the nearest router. The router then append its reading to the end of the uplink packet and forward it to the next router, this process done by a router will be repeated by every router in the network until the packet arrives its destination that is the coordinator node in these experiments.

\section{CONCLUSIONS AND FUTURE WORK}

This paper studies a MAC protocol for wireless sensor networks (WSNs) that focuses on road traffic monitoring applications, which can be implemented on monitoring serious issues like bridges vibration, pollution and gases emissions in tunnels and any factor could affect traffic and drivers health, and for GIS data collection for better practices targeted by Intelligent Transportation Systems(ITSs). The Jennic JN5148 evaluation kit attached with some dedicated sensors is the main used hardware component for building a road monitoring application, that utilizes the proposed $M A C$ method protocol. Furthermore, the proposed protocol is designed to support WSN where its nodes are arbitrary deployed and distributed to form in a linear topology (LWSN). This work demonstrates the validity of the MAC protocol by building a complete end-toend road traffic monitoring system by using 4 nodes deployed in an indoor environment. Regrading the ingoing work, we intend to increase the scalability of the system in order to support more nodes in the wireless sensor network compared to current implementation. Whereas, our target is to extend the proposed MAC scheme to support 20 nodes. In order to achieve this goal we propose to modify the current addressing method; Instead of using the typical 8 bytes for node address, we could just use two bytes for node address. The proposed MAC overcomes the typical cluster tree algorithm [12] by providing reliable communication links and avoiding well-known limitation of the earlier mentioned algorithm. Furthermore, the main features of the proposed MAC are to overcome some of limitations of the Jennic propriety MAC implementation by decreasing the protocol overhead, increasing maximum data packet size, providing a bidirectional communication links and supporting the linear network topology.

\section{REFERENCES}

[1] S. Tarapiah, S. Atalla, and R. AbuHania, "Smart on-board transportation management system using gps/gsm/gprs technologies to reduce traffic violation in developing countries," International Journal of Digital Information and Wireless Communications (IJDIWC), vol. 3, no. 4, pp. 96-105, 2013.

[2] S. Tarapiah and S. Atalla, "Public transportation management system based on gps/wifi and open street maps," International Journal of Advanced Computer Science and Applications (IJACSA), vol. 6, no. 1, pp. 189-194, 2015.

[3] B. M. Velichkovsky, S. M. Dornhoefer, M. Kopf, J. Helmert, and M. Joos, "Change detection and occlusion modes in road-traffic scenarios," Transportation Research Part F: Traffic Psychology and Behaviour, vol. 5, no. 2, pp. 99-109, 2002.

[4] S. Coleri, S. Y. Cheung, and P. Varaiya, "Sensor networks for monitoring traffic," in Allerton conference on communication, control and computing, 2004, pp. 32-40.

[5] S. Y. Cheung, S. C. Ergen, and P. Varaiya, "Traffic surveillance with wireless magnetic sensors," in Proceedings of the 12th ITS world congress, vol. 1917, 2005, p. 173181.

[6] Z. Wang, X. Zha, and X. Qian, "The application and issuse of linear wireless sensor networks," in System Science, Engineering Design and Manufacturing Informatization (ICSEM), 2011 International Conference on, vol. 2. IEEE, 2011, pp. 9-12.

[7] Jennic, "Jennic Website," http://www.jennic.com/, 2010, [Online; accessed 01-April-2015].

[8] R. SOKULLU and E. DEMIR, "Mac layer protocols for linear wireless sensor networks: A survey."

[9] S. Tarapiah, S. Atalla, N. Muala, and S. Tarabeh, "Offline public transportation management system based on gps/wifi and open street maps," in Computational Intelligence, Communication Systems and Networks (CICSyN), 2014 Sixth International Conference on. IEEE, 2014, pp. 182-185. 
[10] S. Tarapiah, S. Atalla, and B. Alsayid, "Smart on-board transportation management system geo-casting featured," in Computer Applications and Information Systems (WCCAIS), 2014 World Congress on. IEEE, 2014, pp. 1-6.

[11] S. Tarapiah, R. AbuHania, I. Hindi, and D. Jamal, "Applying web based gps/gprs ticketing and tracking mechanism to reduce traffic violation in developing countries," in The International Conference on Digital Information Processing, E-Business and Cloud Computing (DIPECC2013). SDIWC - Digital Library, 2013, pp. 102-106.

[12] M. D. Sarr, F. Delobel, M. Misson, and I. Niang, "Automatic discovery of topologies and addressing for linear wireless sensors networks," in Wireless Days (WD), 2012 IFIP. IEEE, 2012, pp. 1-7.

[13] libelium, "Smart Roards," http://www.libelium.com/smart_roads_wsn_ smart_infrastructures/, [Online; accessed 01-April-2015].

[14] N. Semiconductors, "Application Note JenNet Application Template (JN-AN-1061)," http://www.jennic.com/files/support_documentation/ JN-AN-1061-JenNet-ApplicationTemplate.pdf, 2010, [Online; accessed 01-April-2015].

[15] Jennic, "Jennic JN5148-EK010 Evaluation KIT," http://www.jennic. com/files/support_files/JN-UG-3062-JN5148-EK010-User-Guide.pdf, 2010, [Online; accessed 01-April-2015]. 